\title{
3D Microstructure-based finite element modeling of deformation and fracture of $\mathrm{SiCp} / \mathrm{Al}$ composites
}

Jie Zhang ${ }^{\mathrm{a}}$, Qiubao Ouyang ${ }^{\mathrm{a}}$, Qiang Guo ${ }^{\mathrm{a}}$, Zhiqiang $\mathrm{Li}^{\mathrm{a}}$, Genlian Fan ${ }^{\mathrm{a}}$, Yishi Su ${ }^{\mathrm{a}}$, , Lin Jiang ${ }^{\mathrm{b}}$, Enrique J. Lavernia ${ }^{\mathrm{b}}$, Julie M. Schoenung ${ }^{\mathrm{b}}$, Di Zhang ${ }^{\mathrm{a}}$

(a) State Key Laboratory of Metal Matrix Composites, Shanghai Jiao Tong University, 800

Dongchuan Road. Shanghai 200240, China

(b) Department of Chemical Engineering and Materials Science, University of California, Davis, CA 95616, USA

ABSTRACT. The mechanical behavior, with particular emphasis on the damage mechanisms, of SiCp/Al composites was studied by both experiments and finite element analysis in this paper. A 3D microstructure-based finite element model was developed to predict the elasto-plastic response and fracture behavior of a 7vol.\% $\mathrm{SiCp} / \mathrm{Al}$ composite. The 3D microstructure of $\mathrm{SiCp} / \mathrm{Al}$ composite was reconstructed by implementing a Camisizer XT particle size analysis device and a random sequential adsorption algorithm. The constitutive behavior of the elastoplastic-damage in the metal matrix, the elastic-brittle failure for the particle reinforcement, and the traction-separation for interfaces, were independently simulated in this model. The validity of the modeling results were validated by the agreement of the experimental stress-strain curve and the morphology of fracture section with those predicted by the simulation. The visual elasto-plastic deformation process, along with crack generation and propagation was well simulated in this model. The numerical results were used to provide insight into the damage mechanisms of $\mathrm{SiCp} / \mathrm{Al}$ composites, and the effects of interfacial strength and particle strength on material properties were also discussed in detail. 
KEYWORDS: Metal-matrix composites (MMCs), Damage mechanics, Finite element analysis (FEA), Interfacial strength

\section{Introduction}

The deformation and fracture behavior of Particle Reinforced Metal Matrix Composites (PRMMCs), is of great importance in many applications, particularly in the automotive and aerospace industries [1]. In the literature, there are generally three approaches that have been used to study the mechanical behavior of MMCs: experimental approach [2, 3], conventional analytical methods [4] and numerical modeling [5-8], each of which has its distinct advantages and limitations. Specifically, experimental studies are able to give the relationship between the microstructure and macroscopic properties, for example the stress-strain curve. However, they are not able to characterize the actual interactions that occur between the matrix, the reinforcement, and the interfaces during deformation and fracture processes. Conventional analytical methods represent another approach that has been successfully applied to investigate deformation mechanisms in MMCs. For example, Ganesh \& Chawla [9] investigated the elastic behavior of SiCp/Al composites using analytical models and predicted the anisotropy in Young's modulus, which was found to agree well with experimental results. Yet analytical models are unable to accurately predict the plastic deformation and fracture processes in PRMMCs due to the absence of microstructural information in those models [10]. In contrast to analytical and experimental approaches, numerical models can account for microstructural details, and hence can be implemented to 
provide valuable insight into the mechanical properties and fracture mechanisms in PRMMCs.

In the numerical modeling of composite materials, there exist two popular approaches: discrete (distinct) element method (DEM) and finite element method (FEM). An enhanced bond model of DEM (MLPG-Eshelby Method) for heterogeneous materials was developed by Han et al. $[11,12]$ and subsequently used by Ouyang et al. [13] to numerically study the deformation and fracture of PRMMCs. Although the DEM model has a faster convergence rate than FEM, it neglects some important factors that are necessary for the study of deformation and fracture mechanisms in PRMMCs, such as the geometry of the particle, the brittle fracture of the particle and the friction forces following interface debonding. FEM is often used to fully reconstruct the microstructure of a composite material, including the inhomogeneous spatial distribution of particles, the irregular morphology of the particles, and the anisotropy of properties in the particles. A few effective 3D numerical models based on FEM have been proposed to study the mechanical properties of PRMMCs. For example, Chalwa et al. [14] acquired the 3D microstructure of PRMMCs using a serial sectioning process in order to reproduce, visualize, and model it with FEM. Compared to simple shaped (spherical or ellipsoidal) single-particle or multi-particle models [15] and 2D finite element models [16], the 3D microstructure-based approach proved to be more accurate in simulating and investigating both macroscopic and microscopic material behavior. However, in the numerical investigation of PRMMCs mentioned above and in literatures [8, 17-20], the fracture behavior, which is vital for modern design and application of PRMMCs, is always neglected due to the associated complexities.

Although there are previous studies explicitly considering the fracture behavior of composite in 3D [21-23], they are not able to account for the effect of particle 
morphology on the properties of $\mathrm{SiCp} / \mathrm{Al}$ composites, since these finite element models are established based on spherical particle assumption. Meanwhile, the brittle failure of particles was not taken into account in these models. In this work, a 3D microstructure-based finite element model was developed to predict the elasto-plastic response and fracture behavior of PRMMCs. A Camisizer XT particle size analysis device was used to obtain the morphology of the SiC particles, and a random sequential adsorption algorithm was applied to reconstruct the microstructure of this PRMMCs. The process is much simpler than that reported in literature [14] but still has reasonable accuracy. Moreover, in order to study the fracture mechanisms, distinct constitutive behaviors of different phases (elasto-plastic damage for the metal matrix, the elastic-brittle failure for the particle reinforcement, and the traction/shear-separation for the interface) were considered in this model. The numerical model was validated through a comparison between simulation and experiment, including stress-strain curves and the morphology of fracture section. A visible elasto-plastic deformation along with crack generation and propagation was then provided, which is helpful for the understanding of the deformation process and fracture mechanisms in $\mathrm{SiCp} / \mathrm{Al}$. The effects of interfacial strength and particle strength on material properties were also discussed.

\section{Materials and Experimental Procedures}

$\mathrm{SiCp} / \mathrm{Al}$ composites encompass an important class of PRMMCs, and thus a 7vol.\% $\mathrm{SiCp} / 7 \mathrm{~A} 04$ was selected as the model material in this study. In this composite, commercial $\alpha$-SiC particles with an average particle size of $13 \mu \mathrm{m}$ were used as the reinforcement and the engineering 7A04 aluminum alloy was selected as the matrix. The $\mathrm{SiCp} / \mathrm{Al}$ composites was produced by stir casting technique and was extruded into 12 mm-diameter bars with an extrusion ratio of 9:1 and then 10:1. Subsequently, the 
composite bars were heat-treated at $470^{\circ} \mathrm{C}$ for 60 minutes, followed by a water quench [24].

A scanning electron microscope (SEM 515, PHILIPS Co. Ltd., Netherlands) and a transmission electron microscope (TEM, JEM-2100F, JEOL, Japan) were used for the characterization of the microstructure of the composite. The microstructure of the as-fabricated $\mathrm{SiCp} / \mathrm{Al}$ is illustrated in Fig. 1(a), which shows that the $\mathrm{SiC}$ particles are uniformly distributed, and there is no observable porosity in the composite. The particle size in this section is between $10 \mu \mathrm{m}$ and $15 \mu \mathrm{m}$, with an average of about $13 \mu \mathrm{m}$. A magnified rendition in Fig. 1(b) shows the heterogeneous structure (Al matrix, SiC particle and interface) in the composite. In this composite, we did not observe a distinct interface layer under TEM, so a zero thickness of the interface layer was assumed in our model. Uniaxial tensile specimens of $\mathrm{SiCp} / \mathrm{Al}$ composites with a diameter of $5 \mathrm{~mm}$ and gauge length of $25 \mathrm{~mm}$ were machined from the extruded composite samples. Experimental tensile tests were performed using a universal testing machine (AUTO-GRAPHAG-I, Shimadzu Co. Ltd., Japan) with a maximum load of $50 \mathrm{kN}$ at a constant strain rate of $5 \times 10^{-4} \mathrm{~s}^{-1}$, operated at room temperature. Details of the tensile tests can be found in reference [25].

\section{Finite element model}

\subsection{Microstructure reconstruction and boundary conditions}

A 3D representative volume element (RVE) was used to represent the selected composite microstructure in this paper. The morphology of $\mathrm{SiC}$ particles having a polyhedral morphologies, as demonstrated in Fig. 2(a), are clearly observed using SEM. The particle morphologies are relatively irregular and one of the typical particle shapes used in this material is illustrated in Fig. 2(b). Statistical information about the particles was established using a Camisizer XT device (Shanghai, Retsch Co. Lt, China). The 3D structural modeling of SiC particles was then created based on this information. One 
typical particle reproduced using this method is illustrated in Fig. 2(c). Then, an efficient random sequential adsorption algorithm was developed to randomly deposit the $3 \mathrm{D}$ particles without overlapping with each other in the cubes of metal matrix. The geometrical parameters of $\mathrm{SiC}$ particles, such as their size, shape, positions and volume fractions are controllable in this program, which is useful to reproduce PRMMCs reinforced with any type and shape of particles based on the actual geometrical information. Note that the model generated here is a special one, in which no particles cross the cell surface. This is the limitation for using cohesive element in Abaqus to simulate the $\mathrm{SiCp} / \mathrm{Al}$ composite. First, the interface layer generated by cohesive element can't be cut in the Abaqus. Second, the rotational degree was constrained in the loading surface of RVE while the rotational degree was unavailable in Abaqus now.

Three microstructure-based RVE models $(25 \mu \mathrm{m} \times 25 \mu \mathrm{m} \times 25 \mu \mathrm{m}, 50 \mu \mathrm{m} \times 50$ $\mu \mathrm{m} \times 50 \mu \mathrm{m}$ and $75 \mu \mathrm{m} \times 75 \mu \mathrm{m} \times 75 \mu \mathrm{m})$ of the $7 \mathrm{vol} . \% \mathrm{SiCp} / \mathrm{Al}$ composite was recreated using the proposed tool. The average size of the reproduced particles is $13 \mu \mathrm{m}$ which is consistent with the result of particle size analysis. The models with different sizes and the particle distributions in these models were presented in Fig 3. There are respectively 7,14 and 31 particles in these models. In terms of the finite element discretization, the general linear 3D solid elements C3D4 in ABAQUS were used to mesh both the matrix and the $\mathrm{SiC}$ particles. A fine mesh was used $(0.5 \mu \mathrm{m}$ and $0.8 \mu \mathrm{m}$ were used for $\mathrm{SiC}$ and matrix, respectively) to discrete the geometrical model. Moreover, the cohesive element (COH3D8 and COH3D6 in Abaqus element library) was adopted to account for the interface behavior between matrix and particles. Here, a non-conforming cohesive element was used in our model. There are two reasons. One aspect the shape of interface layer is as complex as particle, COH3D6 element has good coordination. Another aspect, the insertion of COH3D6 element is helpful to mesh the 
interface layer with the same mesh size. In the system of SiCp/Al composite, the inner surface of cohesive element ties with the surface of particle, while the outer one ties with the matrix surface which contacts with the relative particle. By this way, the matrix and particles were bonded together, and then directly contact with each other when the cohesive element fails.

These RVE models were generated in the XYZ Cartesian cordinate system. To simulate the tensile process of $\mathrm{SiCp} / \mathrm{Al}$ composite, the boundary conditions were discussed firstly. The periodic boundary conditions are usually preferred for the prediction of mean effective response but cannot provide good estimates of the physical RVE size of random particle reinforced composites. To the problem of large deformation and fracture, the periodic boundary condtions is obviously unacceptable in the embedding models used in this paper. Other four different sets of boundary conditions (static uniform, kinematic uniform and two mixed static-kinematic uiforms) were discussed by Cugnoni [26] in the uniaxial response of a material with random microstructure. After comparsion, two boudary conditions were considered in this paper: the load- and displacement-controlled experiments, in which load or displacement is imposed in the tension-compression direction while the specimen lateral surface is stress-free. These boundary conditions are computationally less expensive than others since they do not impose any constraints on the displacements perpendicular to the load direction. The load-controlled experiment is purely static uniform which is not suitable for the fracture simulation. The displacement one is the mixed static-kinematic boundary conditions which were adopted by researches $[27,28]$ to simulate the behavior of particle reinforced composites. Hence, in this paper, in one hand, a displacement loading of $10 \mu \mathrm{m}$ applied to all models by fixing the displacement of $\mathrm{Y}-\mathrm{Z}$ plane in $\mathrm{X}$ directions, and applying a uniaxial displacement on the parallel $\mathrm{Y}-\mathrm{Z}$ 
plane in the $\mathrm{X}$ direction. The displacement loading of $10 \mu \mathrm{m}$ is a mixed uniform boundary conditions. This displacement loading was imposed in the tensile direction while the cubic domain faces parallel to the tensile direction were stress-free. In the other hand, the rotation degree of the nodes with displacement loading (only the fixed surface and the loading surface of RVE) was constrained along all three axes. In our experience, fixing the rotation degree of the nodes on loading surface is helpful to guarantee the stability of the model after fracture happens. The stress state in the different regions of macroscopic tensile specimen is different and complex. It is difficult to define the real boundary conditions for the RVE, especially at the domain with large deformation and fracture. This paper applied a simple boundary condition and paid specially attention on simulating the deformation and fracture mechanism of SiCp/Al composite.

\subsection{Constitutive behaviors}

In the system of $\mathrm{SiCp} / \mathrm{Al}$ composite, we assume that the elasto-plastic deformation and ductile damage occurs on the matrix, the elastic deformation and brittle failure can takes place in on $\mathrm{SiC}$ particles, and the traction-separation behavior happens on interface according to their mechanical properties. To predict the elasto-plastic response of 7A04 Al matrix, the Ludwik law was used, where the flow stress can be expressed as:

$\sigma=\sigma_{0}+K\left(\bar{\varepsilon}^{p}\right)^{n}$

in which $\sigma$ is the flow stress, $\sigma_{0}$ is the yield stress, $\bar{\varepsilon}^{p}$ is the equivalent plastic strain, $K$ and $n$ are the parameters of work-hardening. Material parameters for 7A04 $\mathrm{Al}$ alloy were obtained from the uniaxial tensile tests conducted in Ref. [25], and are as follows: $\sigma_{0}=503 \mathrm{MPa}, K=193 \mathrm{MPa}$, and $n=0.45$. To model the fracture behavior of aluminum, a ductile criterion was used to predict the onset of damage. Moreover, a 
damage evolution law was applied to describe the rate of degradation of the material stiffness [29]. A scalar damage variable $D$ associated with plastic strain having a value from 0 to 1 was defined to represent degradation of the material stiffness. In this model, damage initiates when the plastic strain to fracture $\bar{\varepsilon}_{f}^{p}$ equals to 0.11 , and the total plastic strain of the matrix, from the initiation of damage to its completion, was assumed to be 0.001. The configuration of damage evolution is important in re-establishing equilibrium after an element failure. A very small plastic strain is used here to do some efforts on this consideration. However, it is better to use the VUMAT to implement a damage evolution (Chaboche model [30, 31] for example) based on the Continue Damage Mechanism (CDM) in Abaqus for the ductile matrix. Other parameters needed in the modelling of 7A04 aluminum include the density $\left(2.7 \mathrm{~g} / \mathrm{cm}^{3}\right)$, Young's modulus (71 GPa), and the Poisson's ratio (0.33) [21]. Note that, although the damage evolution was implemented by a plastic strain of 0.001 which is much smaller than the $\bar{\varepsilon}_{f}^{p}$ defined here, a mesh size effect should be investigated or a mesh size independent constitutive model should be adopted to consider the mesh dependent behavior.

To describe the elastic response and brittle fracture behavior of $\mathrm{SiC}$ particles, we used the "brittle cracking model" of ABAQUS/Explicit. It is a cracking model for concrete and other brittle materials, such as ceramics or brittle rocks [27, 28]. In our model, both Mode I and Mode II failure mechanisms were considered. A simple Rankine criterion was first used to detect crack initiation, which assumes that the crack forms when the maximum principal tensile stress exceeds the tensile strength of the $\mathrm{SiC}$ particles $\left(\sigma_{f}^{p}\right)$. The Mode I fracture was considered in the crack initiation, while Mode 
II shear behavior was taken into account in postcracked behavior to describe the reduction of shear modulus for shear components after crack initiation:

$D\left(e^{c r}\right)=\alpha\left(e^{c r}\right) G$

where $e^{c r}$ is the fracture strain which is a part of total strain, $G$ is the shear modulus of the uncracked $\mathrm{SiC}$ particles and $\alpha\left(e^{c r}\right)$ is a material parameter written as :

$\alpha\left(e^{c r}\right)=\frac{\rho}{1-\rho}$, and $\rho\left(e^{c k}\right)=\left(1-\frac{e^{c k}}{e_{\max }^{c k}}\right)^{p}$

where $p$ and $e_{\max }^{c k}$ are material parameters used to control the shear retention. Because of the absence of experimental data on SiC particles, these parameters were numerically extrapolated from fitting the tensile force-displacement curve of $7 \%$ $\mathrm{SiCp} / \mathrm{Al}$ composite, as $\sigma_{f}^{p}=1000 \mathrm{MPa}, e_{f}^{c r}=0.02, \quad e_{\max }^{c r}=0.2$ and $p=2$. Other parameters needed in the modeling for $\mathrm{SiC}$ particle include the density $\left(3.2 \mathrm{~g} / \mathrm{cm}^{3)}\right.$, the Young's Modulus (427 GPa) and Poisson's ratio (0.17) [25].

An element-based cohesive approach is adopted to predict the traction-separation behavior of interface. It assumes that the damage initiates at the interface when a quadratic interaction function involving the nominal stress ratios reaches a value of one, as:

$$
\left\{\frac{\left\langle t_{n}\right\rangle}{t_{n}^{0}}\right\}^{2}+\left\{\frac{t_{s}}{t_{s}^{0}}\right\}^{2}+\left\{\frac{t_{t}}{t_{t}^{0}}\right\}^{2}=1
$$

Here, $t_{n}^{0}, t_{s}^{0}$ and $t_{t}^{0}$ represent the peak values of the nominal stress (interfacial strength). A scalar damage variable $D_{1}$ is defined to represent the overall damage of interfacial layer once the corresponding initiation criterion is satisfied. Assuming linear softening, an evolution of damage variable $D_{l}$ is introduced in Abaqus: 
$D_{1}=\frac{\delta_{m}^{f}\left(\delta_{m}^{\max }-\delta_{m}^{0}\right)}{\delta_{m}^{\max }\left(\delta_{m}^{f}-\delta_{m}^{0}\right)}$

where, $\delta_{m}^{\max }$ refers to the maximum value of the effective displacement, $\delta_{m}^{f}$ is the effective displacement at complete failure and $\delta_{m}^{0}$ is the effective displacement at the initiation of damage. The effective displacement at complete failure $\delta_{m}^{f}$ equals to 0.00025 , which is deduced from the fracture energy obtained from Ref. [25]. The detailed measurement of interfacial strength and its effects on material responses were numerically studied in the following section.

Although some important aspects of deformation and fracture behavior in PRMMCs were considered in the proposed FE model, there are some limitations and assumptions. Actually, to keep the problem tractable, defects in the matrix, particle or interfaces were neglected in the 3D modeling of the composite microstructure. Also, average values for the interfacial strength and particle strength that can fit the experimental stress-strain response of the bulk composite were used in this study.

\section{Numerical results and discussion}

\subsection{Numerical studies on the representative volume element}

The predicted stress-strain behavior for RVE with different sizes was shown in Fig. 4, where it is clearly shown that the elastic modulus was not significantly affected by the change in model size, while the yield stress increases and the elongation decreases with increasing size of RVE. However, the stress-strain response was relatively unchanged at $50 \times 50 \times 50(\mu \mathrm{m})$ and above. Analyzing the particle fracture, one can find that the fracture ratio of particle were $57.14 \%, 30 \%$ and $29.03 \%$ for $25 \times 25 \times 25$ $(\mu \mathrm{m}), 50 \times 50 \times 50(\mu \mathrm{m})$ and $75 \times 75 \times 75(\mu \mathrm{m})$ respectively, indicating that the fracture ratio of particle was relatively unchanged for $50 \times 50 \times 50(\mu \mathrm{m})$ and above. Therefore, 
in subsequent simulation and analysis, only $50 \times 50 \times 50(\mu \mathrm{m}) \mathrm{RVE}$ were studied to reveal the mesh size and the microstructure variability effects of the model prediction.

Three models with different mesh size group (particle mesh size, matrix mesh size) were considered to study the mesh size effect: a coarse mesh $(1.0 \mu \mathrm{m}, 1.6 \mu \mathrm{m})$, a fine mesh $(0.5 \mu \mathrm{m}, 0.8 \mu \mathrm{m})$ and a very fine mesh $(0.3 \mu \mathrm{m}, 0.5 \mu \mathrm{m})$. The tensile responses of these models are presented in Fig. 5. One can find that the crack paths in Fig. 5(a), (b) and (c) were similar and the stress-strain curves in Fig .5(d) have no obvious difference in yield strength, ultimate tensile strength and uniform elongation when the mesh size is finer than the group of $(0.5 \mu \mathrm{m}, 0.8 \mu \mathrm{m})$.

Further, to study the model variability, three models were randomly reconstructed for the $50 \times 50 \times 50(\mu \mathrm{m})$ RVE model with the mesh size $(0.5 \mu \mathrm{m}, 0.8 \mu \mathrm{m})$. The deformation and fracture behavior of the three models is compared in Fig. 5(e), which shown almost identical deformation and fracture behavior. Thus, we concluded that the model with the size of $50 \times 50 \times 50(\mu \mathrm{m})$ can serve as a RVE, and the models with mesh size finer than $(0.5 \mu \mathrm{m}, 0.8 \mu \mathrm{m})$ can reach an acceptable convergence results of the composite microstructure examined here.

\subsection{Model validation}

We firstly validated the numerical model by comparing the microstructure-based model predictions with the experimental response. Tab. 1 summarizes the experimental and numerical mechanical properties of the composite. These values were obtained from the three models with the same size of $50 \mu \mathrm{m} \times 50 \mu \mathrm{m} \times 50 \mu \mathrm{m}$. The stress-strain curves for these models are shown in Fig. 5-(e). The elastic modulus and its standard deviation are all derived from these curves. Then, we chose the middle stress-strain curve in Fig. 5-(e) to compare with the average experiment value. Fig. 6 presents both the experimental and the simulation-based stress-strain curves for $7 \mathrm{vol} \% \mathrm{SiCp} / \mathrm{Al}$ composite. One can observe that the elastic modulus, the yield stress, the tensile 
strength and the elongation of the composite predicted by the proposed finite element model agree well with the experimental values.

It is necessary to explain that the predicted elastic modulus is close to the Hashin-Shtrikman's upper bounds. This is due to the processes of the preparation of $\mathrm{SiCp} / \mathrm{Al}$ composite. As we mentioned in the manuscript in section 2, the SiCp/Al composites was produced by stir casting technique and was extruded into 12 mm-diameter bars with an extrusion ratio of 9:1 and then 10:1. Subsequently, the composite bars were heat-treated at $470^{\circ} \mathrm{C}$ for 60 minutes, followed by a water quench. Hence, the matrix is stiffer after these processes than that before material preparation. In the other hand, the $\mathrm{SiC}$ particle is also not as stiffer as the $\mathrm{SiC}$ that we test experimentally. In our experience, there are defects in the $\mathrm{SiC}$ particle which has severely affected the stiffness of $\mathrm{SiC}$ in MMC. In this paper, we predict the true properties of $\mathrm{SiCp} / \mathrm{Al}$ composite. The properties of matrix and $\mathrm{SiC}$ particles were tested using the specimens that with the same heat-treatment and machining processes. Donc, in this paper, the experimental results and the numerical results are all shown that the elastic modulus of our $\mathrm{SiCp} / \mathrm{Al}$ is close to the upper Hashin-Shtrikman bounds.

A visible stress and strain evolution, as well as the crack generation and propagation inside the composite during tensile loading are illustrated in Fig. 7 and Fig. 8 , respectively, where the sub-figures correspond to the different stages $(a, b, c, d)$ on the stress-strain curve, as indicated in Fig. 6.

In this simulation, we numerically identified the interfacial strength and particle strength. We first identified the interfacial strength through fitting the initial stage (before the fracture of particle) of experimental stress-strain curve since the debonding of interface happens from the very beginning of the composite deformation. As shown in Fig. 6, the predicted stress-strain curve with an interfacial strength value of 400MPa 
is in good agreement with the experimental results in this initial stage. This value is also consistent with published results [32] for an average particle size of $13 \mu \mathrm{m}$ in a SiCp/Al composite. Then, the particle strength is numerically identified through considering the elongation and the particle fracture ratio of composite. It is clearly demonstrated that the predicted stress-strain curve with a particle strength value of $1000 \mathrm{MPa}$ is in good agreement with the experimental results in elongation. Also, the SEM micrograph in Fig. 9 (a) shows the morphology of an actual fracture section, where three types of failure can be observed: ductile fracture of metal matrix (red region), brittle failure of $\mathrm{SiC}$ particles (black arrow) and debonding of the interface (yellow arrow). In the proposed numerical model, these fracture modes were well predicted as presented in Fig. 9 (b) and the fracture ratio of particle is about $30 \%$ which is consistent with the ratio observed in the experimental fracture sections.

Therefore, the observations provide support to the validity of the constitutive models selected for the various phases in the $\mathrm{SiCp} / \mathrm{Al}$ composite, and suggest that the proposed numerical model can indeed be applied to predict not only the stress-strain response but also the fracture behavior.

\subsection{Effect of interfacial strength and particle brittle failure}

To study the effect of interfacial strength, three models with different interfacial strength values (200 MPa, $400 \mathrm{MPa}$ and $600 \mathrm{MPa}$ ) were investigated. In these models, the $\mathrm{SiC}$ strength is fixed to $1000 \mathrm{MPa}$. The stress-strain curves with different interfacial strength were presented in Fig. 10. One can find that the weaker interfaces promote easier interface debonding, which decreases the stiffness and strength while increases the total elongation. One can also find that a stronger interface can give rise to the further brittle fracture of particles, which decreases the elongation of composite.

In addition, to study the effect of particle strength, three models with different particle strength values (2000 $\mathrm{MPa}, 1000 \mathrm{MPa}$ and $800 \mathrm{MPa}$ ) were investigated. In 
these models, the interfacial strength is fixed to $400 \mathrm{MPa}$. As shown in Fig. 11, the numerical results suggest that the magnitude of the particle strength has effect on the overall composite strength. What is more, when particle strength reaches to $2000 \mathrm{MPa}$, the brittle failure of particles do not occur, giving rise to an increase of both composite's strength and elongation. On contrast, lower particle strength leads to early brittle failure, which decreases not only the composite's strength, but also the elongation. With further analysis of these fractured particles, two fracture modes can be identified: tensile fracture in Fig. 12 (a), and shear fracture in Fig. 12(b). It is not unusual to observe the tensile fracture of particles during tensile testing of PRMMCs. However, shear fracture also appears due to the presence of a compressive stress, which is important and should not be neglected in the analysis of deformation.

In the previous investigations, the determination of representative volume element is under the assumption of elastoplastic composite $[10,14,25,33,34]$. In practice, beside the reinforcement of particle, the tensile/shear-separation of interface, the fracture of particle and the stress state of matrix are three key factors which can affect the dynamic response of composite. Meanwhile, these effects will work at the beginning of the deformation of composite and they are related to the complexity of particles (the shape, the distribution and so on - these factors cause the stress concentration). One can also find that the representativeness of the volume elements for $\mathrm{SiCp} / \mathrm{Al}$ composite is different when the fracture behavior is considered. Hence, it is worth to do more research in determining the $\mathrm{RVE}$ for $\mathrm{SiCp} / \mathrm{Al}$ in the future.

\section{Conclusions}

A 3D microstructure-based finite element model was developed to predict the elasto-plastic response and fracture behavior of a 7vol.\% SiCp/Al composite. The validity of the modeling results were justified by the agreement of the experimental stress-strain curve and the fracture surface morphology with those predicted by the 
simulation. A visible process of elasto-plastic deformation along with crack generation and propagation was described, which is helpful for the study of deformation and fracture mechanisms in SiCp/Al composites. Some salient conclusions from our work are summarized as follows: (1) tensile fracture in the investigated 7\%vol.SiCp/Al composite is initiated by particulate fracture and interface debonding as a result of stress concentration. Void nucleation caused by either cracking or debonding reduces the load capacity of the composite, and accelerates the damage accumulation process; (2) Weaker interfaces promote easier interface debonding, decrease the composite's stiffness and strength, but increase the elongation. (3) Lower particle strength leads to earlier brittle failure, which decreases not only the composite's strength but also its uniform elongation upon tensile loading.

\section{Acknowledgments}

The authors sincerely acknowledge the financial supports by National Basic Research “973” Program (No.2012CB619600), National High-Tech R\&D “863” Program (No.2012AA030311), the Science \& Technology Committee of Shanghai Municipality (No. 13PJ1404000 and No.14DZ2261200), the Office of Naval Research (ONR) under the guidance of Dr. Lawrence Kabacoff (ONR N00014-12-1-0237).

\section{References}

[1] D. Zhang, G. Zhang, Z. Li, Materials China, 29 (2010) 1-7.

[2] P. Hruby, S.S. Singh, J.J. Williams, X. Xiao, F. De Carlo, N. Chawla, International Journal of Fatigue, 68 (2014) 136-143.

[3] C. Yang, Y. Zong, Z. Zheng, D. Shan, Materials Characterization, 96 (2014) 84-92.

[4] Z. Hashin, S. Shtrikman, Journal of the Mechanics and Physics of Solids, 11 (1963) 127-140.

[5] X.Q. Xu, D.F. Watt, Acta Materialia, 44 (1996) 4501-4511.

[6] J.-T. Zhang, L.-S. Liu, P.-C. Zhai, Z.-Y. Fu, Q.-J. Zhang, Composites Science and Technology, 67 (2007) 2775-2785.

[7] J.J. Williams, J. Segurado, J. Llorca, N. Chawla, Materials Science and Engineering: A, 557 (2012) 113-118.

[8] H. Qing, Computational Materials Science, 89 (2014) 102-113.

[9] V.V. Ganesh, N. Chawla, Materials Science and Engineering A, 391 (2005) 342-353.

[10] N. Chawla, K.K. Chawla, J Mater Sci, 41 (2006) 913-925.

[11] Z.D. Han, S.N. Atluri, CMES: Computer Modeling in Engineering \& Sciences, 97 (2014) 119-237. [12] Z.D. Han, S.N. Atluri, CMES: Computer Modeling in Engineering \& Sciences, 97 (2014) 1-34. 
[13] Q. Ouyang, D. Zhang, X. Zhu, Z. Ha, CMC: Computers, Materials \& Continua, 41 (2014) 37-53.

[14] N. Chawla, R.S. Sidhu, V.V. Ganesh, Acta Materialia, 54 (2006) 1541-1548.

[15] W. Han, A. Eckschlager, H.J. Böhm, Composites Science and Technology, 61 (2001) 1581-1590.

[16] G. Tursun, U. Weber, E. Soppa, S. Schmauder, Computational Materials Science, 37 (2006) 119-133.

[17] P. Gudlur, A. Muliana, M. Radovic, Composites Part B: Engineering, 58 (2014) 534-543.

[18] B. McWilliams, J. Dibelka, C.F. Yen, Materials Science and Engineering: A, 618 (2014) 142-152.

[19] P.B. Pawar, A.A. Utpat, Procedia Materials Science, 6 (2014) 1150-1156.

[20] Y. Su, Z. Li, L. Jiang, X. Gong, G. Fan, D. Zhang, Materials Science and Engineering: A, 614 (2014) 273-283.

[21] J. Llorca, J. Segurado, Materials Science and Engineering: A, 365 (2004) 267-274.

[22] L.L. Mishnaevsky Jr, Acta Materialia, 52 (2004) 4177-4188.

[23] C.R. Dandekar, Y.C. Shin, Composites Part A: Applied Science and Manufacturing, 40 (2009) 1231-1239.

[24] Q.B. Ouyang, W.L. Wang, G.D. Zhang, D. Zhang, R.X. Li, Mater Sci Forum, 4 (2007) 546-549.

[25] Y. Su, Q. Ouyang, W. Zhang, Z. Li, Q. Guo, G. Fan, D. Zhang, Materials Science and Engineering: A, 597 (2014) 359-369.

[26] J. Cugnoni, M. Galli, in: Computer Modeling in Engineering and Sciences, 2010, pp. 165-186.

[27] J. Cugnoni, M. Galli, Computer Modeling in Engineering and Sciences, 66 (2010) 165-186.

[28] M. Galli, J. Cugnoni, J. Botsis, European Journal of Mechanics - A/Solids, 33 (2012) 31-38.

[29] Y. Bao, T. Wierzbicki, Engineering Fracture Mechanics, 72 (2005) 1049-1069.

[30] J.L. Chaboche, Nuclear Engineering and Design, 79 (1984) 309-319.

[31] J. Lemaitre, Computer Methods in Applied Mechanics and Engineering, 51 (1985) 31-49.

[32] Y.H. TENG, J.D. BOYD, COMPOSITES, 25 (1994) 906-912.

[33] J.B. Ferguson, X. Thao, P.K. Rohatgi, K. Cho, C.-S. Kim, Scripta Materialia, 83 (2014) 45-48.

[34] I. Alfonso, I.A. Figueroa, J.M. Sierra, M. Abatal, G. Gonzalez, V. Rodriguez-Iglesias, A. Medina-Flores, J.E. Flores, Computational Materials Science, 69 (2013) 304-310. 
Tab. 1 Mechanical properties of 7\%vol.SiCp/Al composite: experiment and numerical.

\begin{tabular}{|c|c|c|c|c|c|}
\hline & $\mathrm{E}(\mathrm{GPa})$ & $\sigma_{0}(\mathrm{MPa})$ & $\sigma_{\text {uts }}(\mathrm{MPa})$ & $\varepsilon_{\mathrm{f}}$ & Specimens \\
\hline Experiment & $84.2 \pm 0.7$ & $528.1 \pm 4.1$ & $568.6 \pm 1.3$ & $0.0282 \pm 0.0011$ & 3 \\
\hline Numerical & $84.9 \pm 1.5$ & $534.0 \pm 5.7$ & $575.0 \pm 5.7$ & $0.0301 \pm 0.0019$ & 3 \\
\hline
\end{tabular}


Fig. 1 Microstructure of SiCp/Al composites: (a) the SEM image of microscopic characteristics and (b) the TEM image of particle-Al interface.

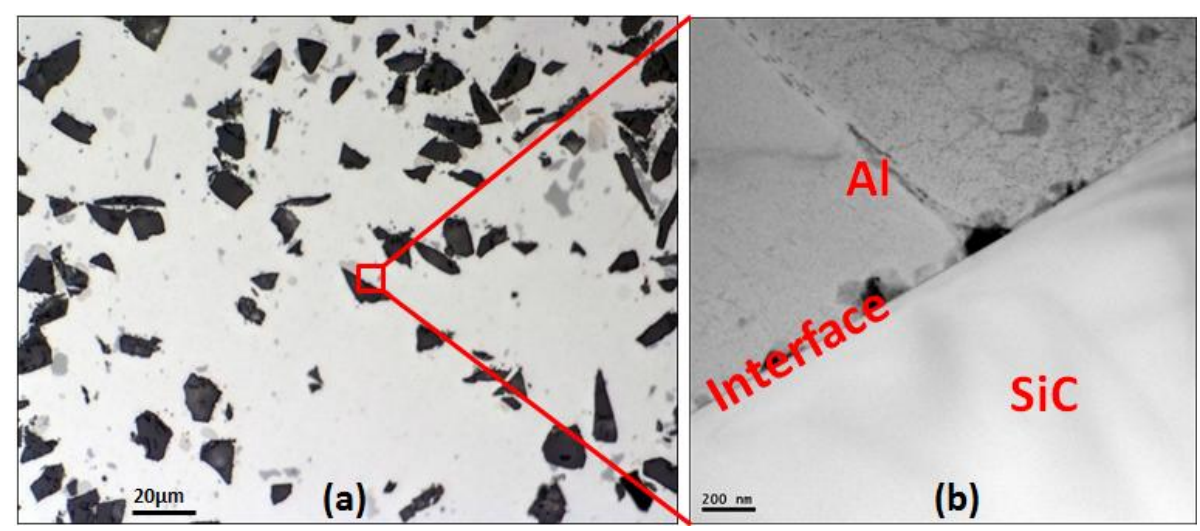

Fig. 2 Morphology of actual SiC particles and their modeling: (a) SiC particles for size and shape analysis; (b) The shape and size of one typical particle, and (c) a typical reproduced particle.
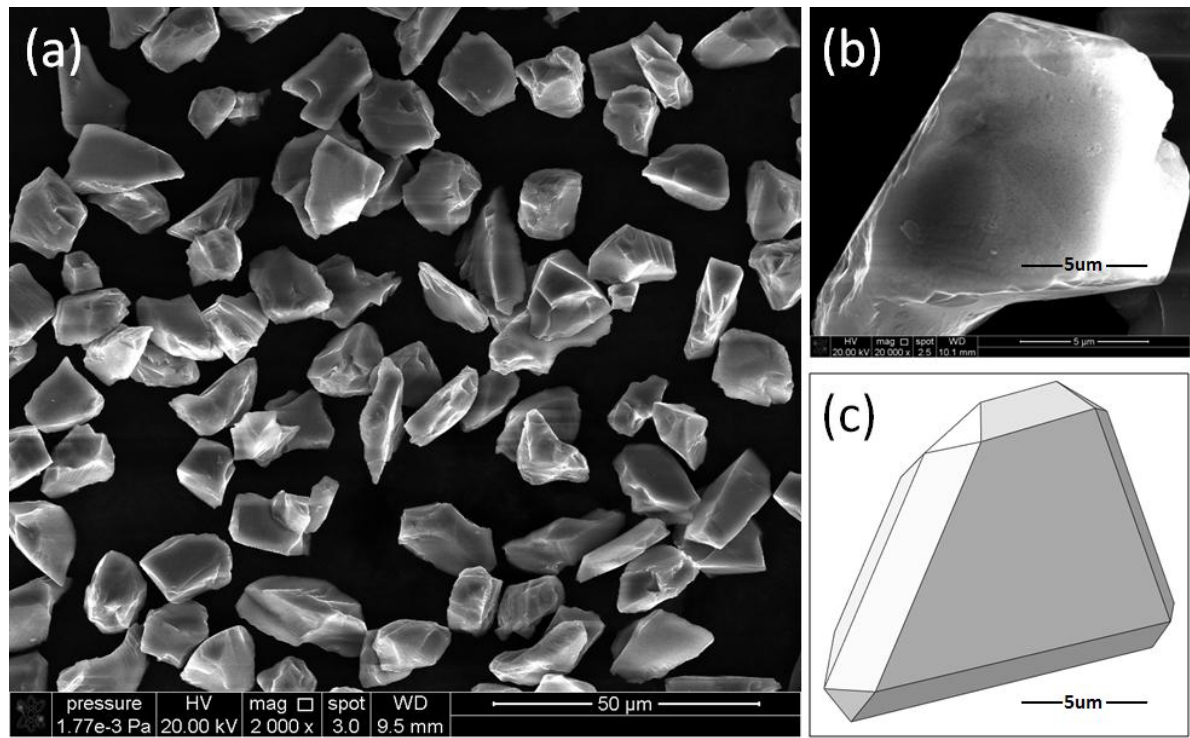

Fig. 3 Models with various sizes used to establish representative volume and the particle distributions: (a) $25 \times 25 \times 25(\mu \mathrm{m})$, (b) $50 \times 50 \times 50(\mu \mathrm{m})$, and (c) $75 \times 75 \times 75(\mu \mathrm{m})$.

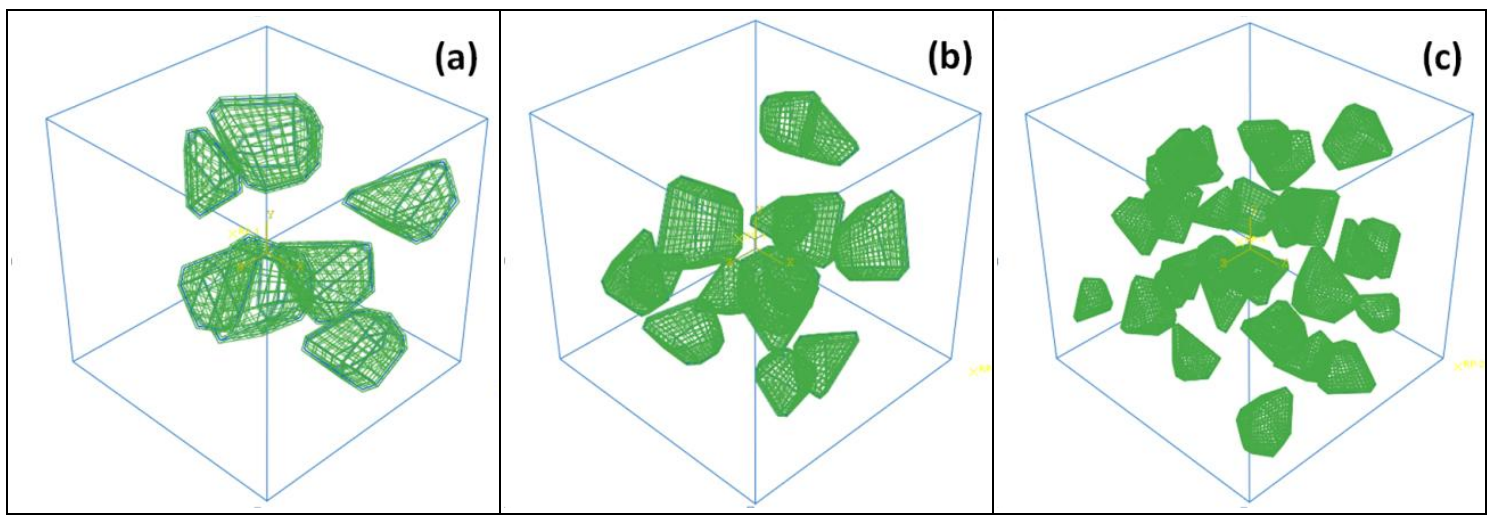


Fig. 4 The tensile stress-strain responses of models with different RVE sizes and the fracture of particles in these RVE models: (a) $25 \times 25 \times 25(\mu \mathrm{m})$, (b) $50 \times 50 \times 50(\mu \mathrm{m})$, and (c) $75 \times 75 \times 75$ $(\mu \mathrm{m})$. The averge particle size in these models is $13 \mu \mathrm{m}$.

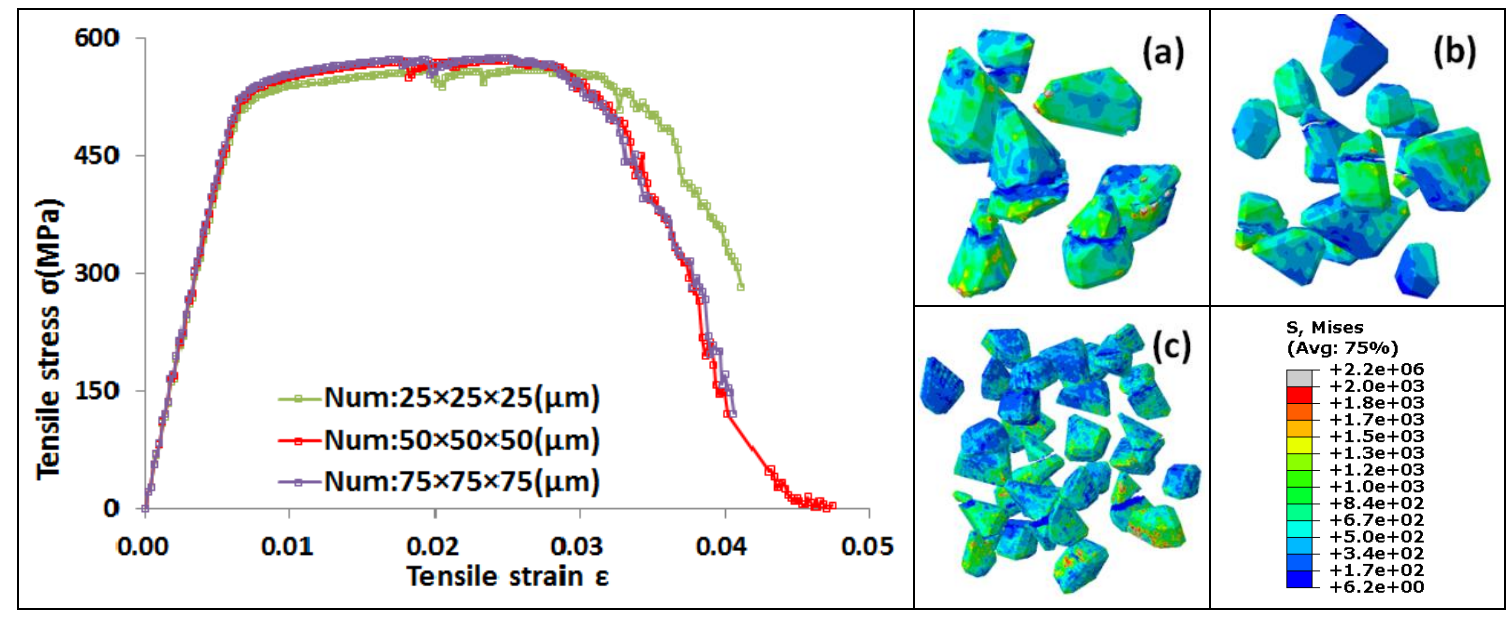

Fig. 5 Study on the tensile response of RVE finite element model with the size of $50 \times 50 \times 50(\mu \mathrm{m})$ : equivalent plastic strain distribution and crack propagation in the model of coarse mesh (a), fine mesh (b) and very fine mesh (c); the mesh size effect (d); and model variation (e).

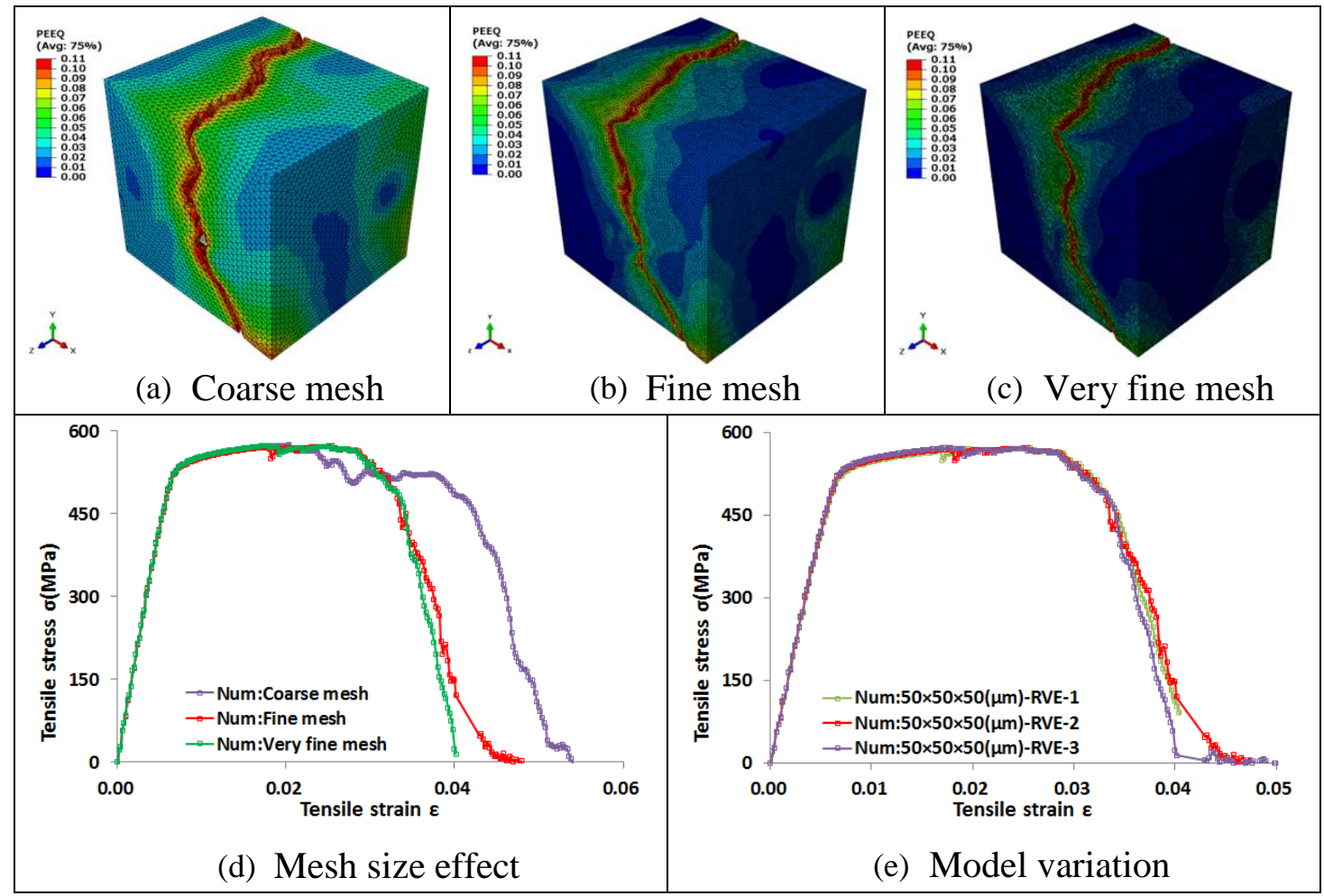


Fig. 6 Comparison of tensile stress-strain curves for 7vol.\% SiCp/Al composites: experiment and simulation.

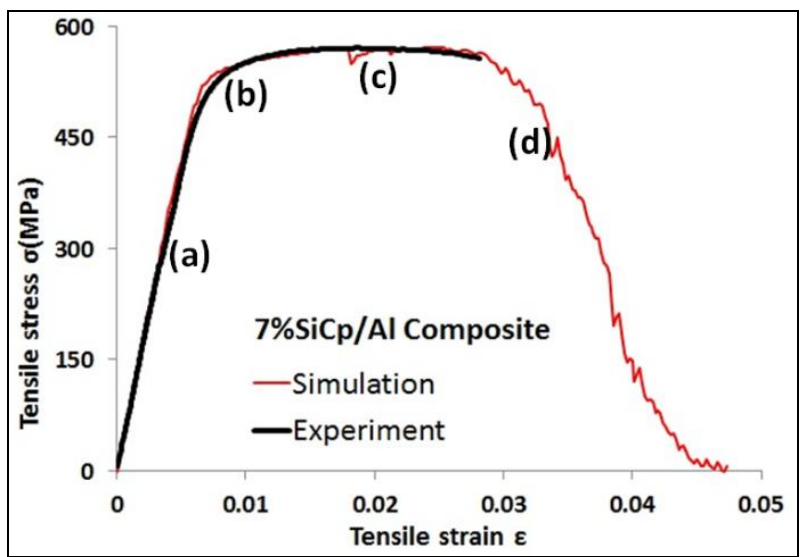

Fig. 7 Stress evolution (Mises yield stress: MPa) and crack initiation and propagation inside the composite during tensile deformation

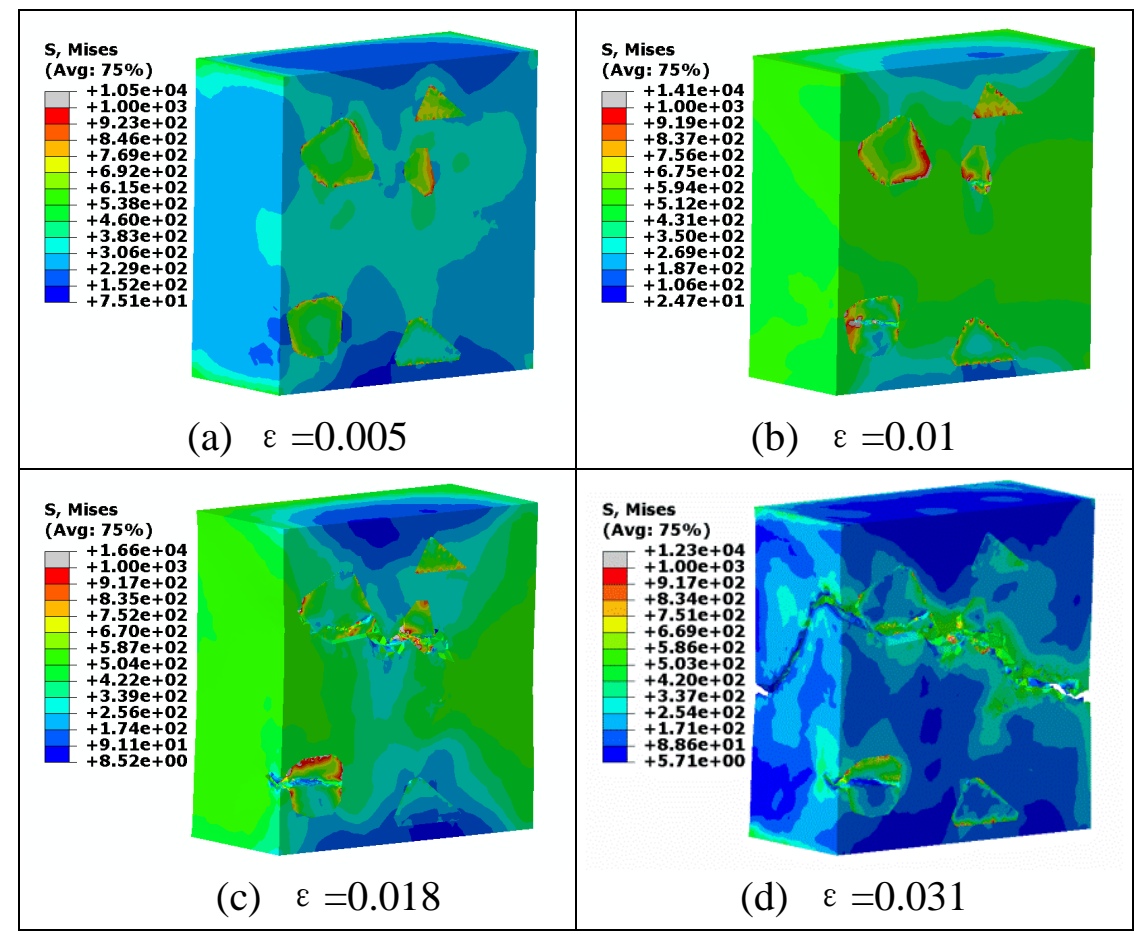


Fig. 8 Equivalent plastic strain evolution and crack generation and propagation inside the composite during tensile deformation: the plastic strain is zero at initiation (a), and it increases to 0.01 and 0.018 from (b) to (c). A major crack passing through the entire volume element forms at $\varepsilon=0.031$ [stage (d)].

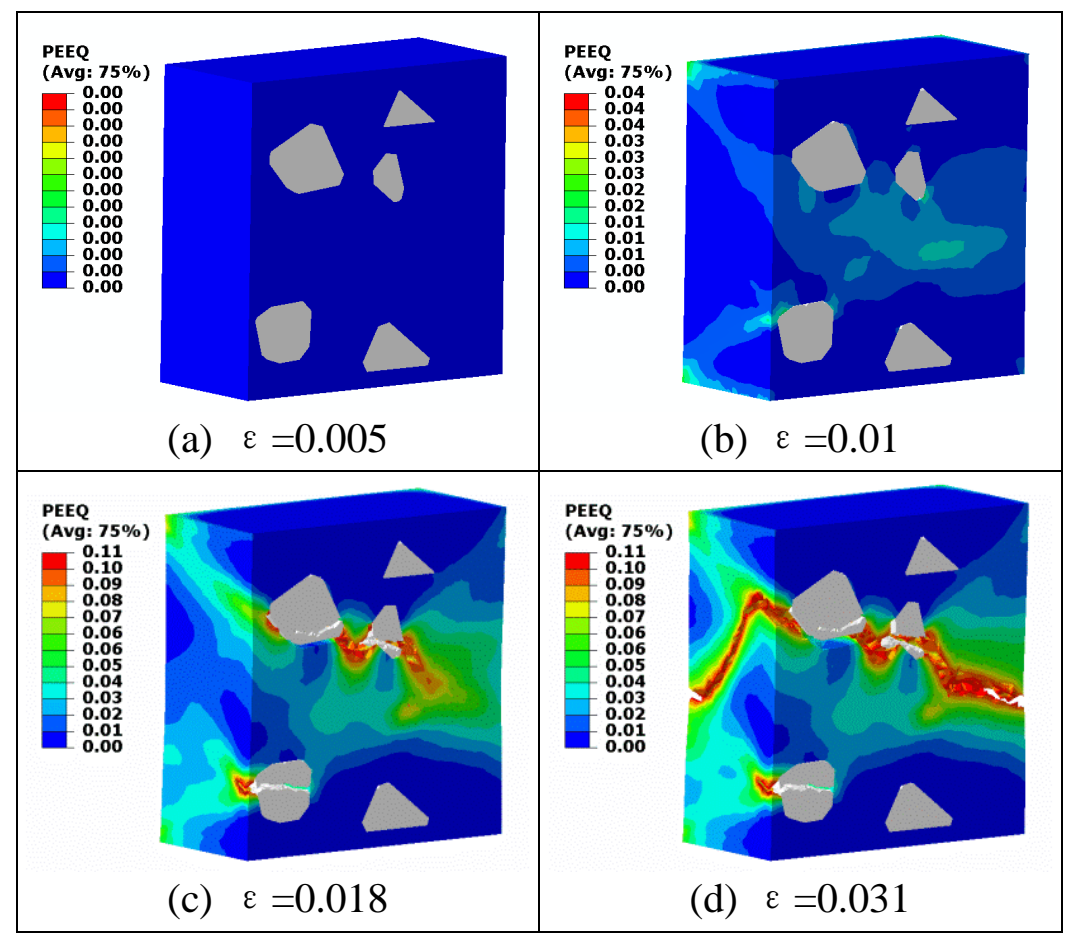

Fig. 9 Morphology of the fracture section of the composite: (a) experiment and (b) numerical model.
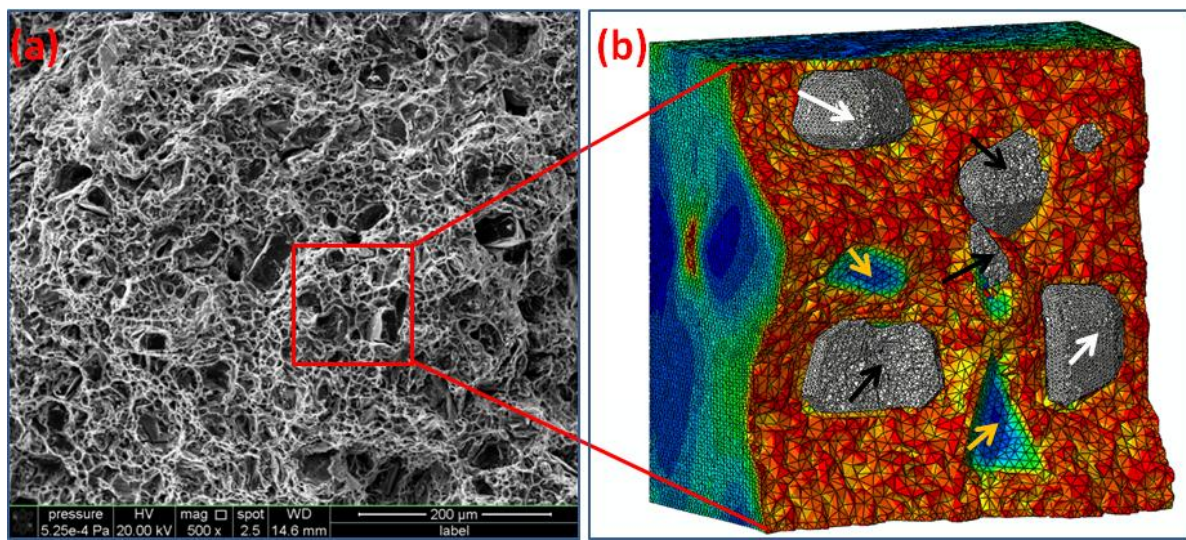
Fig. 10 Numerical studies on the effect of interface strength on the stress-strain responses of the composite: the interface strength of $400 \mathrm{MPa}$ fits well the experiment while the lower interface strength (200MPa) decreases the material stiffness and the higher interface strength (600MPa) leads to earlier fracture of composite.

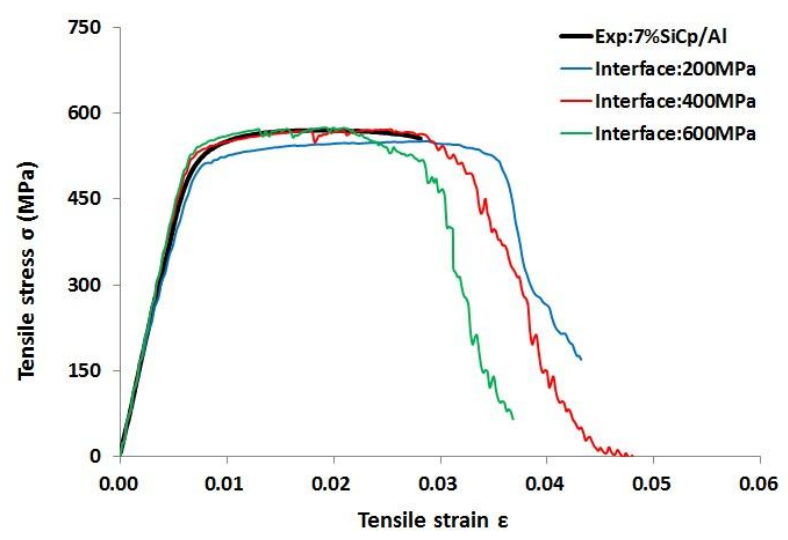

Fig. 11 Stress-Strain responses of different particle strength: the particle strength of 1000MPa fits well the experiment while the lower particle strength (800MPa) leads to earlier fracture of composite and the particles are not fractured in the model with higher particle strength (2000MPa) .

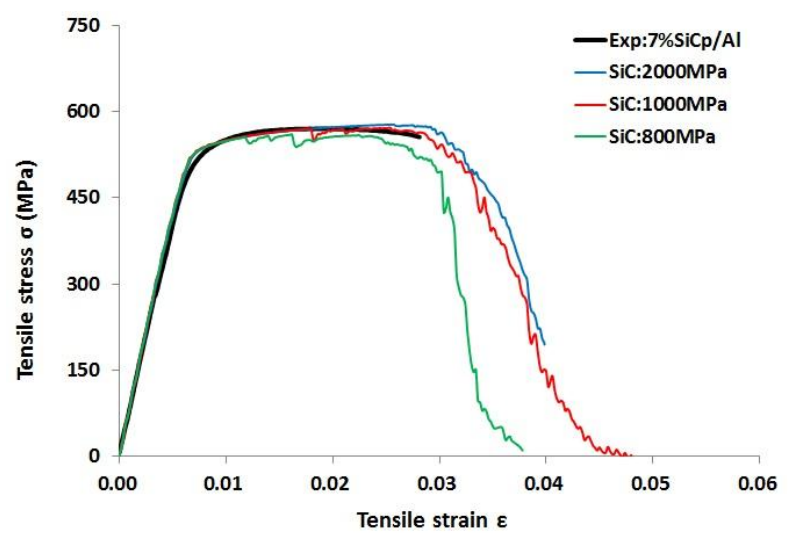

Fig. 12 Fracture of particles in the tensile process of $7 \mathrm{vol} \% \mathrm{SiCp} / \mathrm{Al}$ composite: (a) tensile fracture, (b) shear fracture.

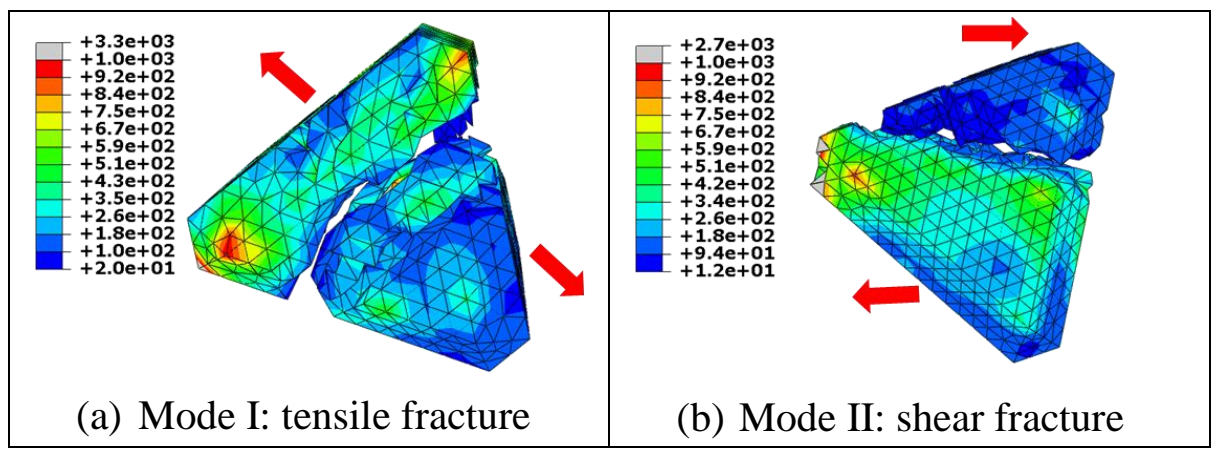

